\title{
Optical design, challenges, and development of the METimage instrument
}

Gabriel Pellegrini

Gabriel Pellegrini, "Optical design, challenges, and development of the METimage instrument," Proc. SPIE 11852, International Conference on Space Optics - ICSO 2020, 118520P (11 June 2021); doi: 10.1117/12.2599172

SPIE Event: International Conference on Space Optics - ICSO 2021, 2021, Online Only 


\section{International Conference on Space Optics-ICSO 2020}

Virtual Conference

30 March-2 April 2021

Edited by Bruno Cugny, Zoran Sodnik, and Nikos Karafolas
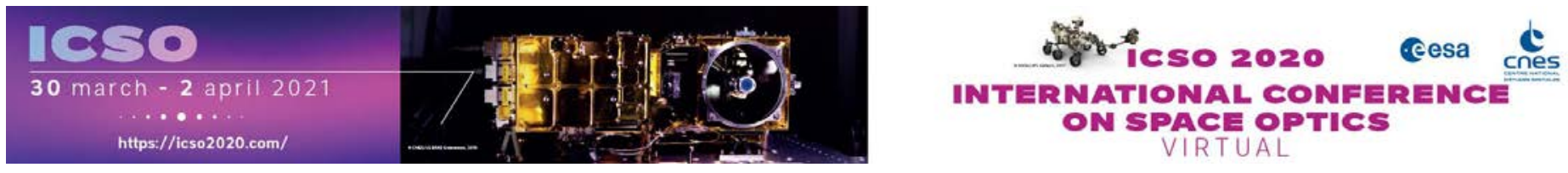

\section{Optical design, challenges, and development of the METimage instrument}

\section{Cesa isoporecestings denes}




\title{
Optical design, challenges and development of the METimage instrument
}

\author{
Gabriel Pellegrini \\ Airbus Defence and Space $\mathrm{GmbH}$, Claude Dornier Strasse, \\ 88090 Immenstaad am Bodensee, Germany
}

\begin{abstract}
METimage is a cross-purpose multi-spectral optical imaging instrument dedicated to operational meteorology, oceanography, and climate applications with medium resolution in 20 spectral bands with wavelengths ranging from 0.4 to $13.5 \mu \mathrm{m}$. The instrument operates onboard MetOp Second Generation satellite A, which is placed in a Sun-synchronous polar orbit with an average altitude of $830 \mathrm{~km}$.

Firstly, we will provide an overview of the optical architecture of the METimage instrument, detailing its specificities:

- In-beam scanner mirror with a cylindrical curvature,

- TMA telescope with very low distortion,

- 5-mirror all SiC derotator,

- Dichroic beamsplitters working from blue to far infrared,

- Cryogenic filters and relay optics for infrared imaging.

Secondly, the key optical design drivers will be described and explained, together with the solutions that have been put in place to address these demands:

- Stringent need on co-registration leading to a very low distortion on the telescope design, and the implementation of masks at the infrared focal planes,

- Pupil imaging and calculation of pupil diffraction generated by the infrared field masks,

- Straylight requirements leading to specific baffling, tight roughness and particulate contamination requirements, careful handling of ghosts and associated post-processing,

- Polarisation sensitivity leading to the specific scanner and derotator combination, and development of optical coatings.

Finally, our current development state will be briefly summarized:

- Successful instrument CDR confirming the maturity of the technical status,

- Design and ongoing manufacturing of the subsystems, focusing on the optical components.
\end{abstract}

Keywords: Multi-spectral imager, METimage, MetOp SG, Optical design 


\section{INTRODUCTION}

This paper gives a brief description of the optical design of the METimage instrument and its subsystems. An insight of the main performance parameters and design drivers is described, as well as the development status of the hardware.

\section{OPTICAL ARCHITECTURE OF THE INSTRUMENT}

\subsection{Instrument concept}

\section{Principle of operation}

METimage is a passive imaging spectro-radiometer, capable of measuring thermal radiance emitted by the Earth and solar backscattered radiation in 20 spectral bands from 0.4 to $13.5 \mu \mathrm{m}$.

The instrument achieves global coverage with $500 \mathrm{~m}$ square pixels by continuous scanning orthogonal to the flight direction.

\section{Scanning principle}

The METimage instrument is designed to cover a large across track swath of around $2670 \mathrm{~km}$ with high spatial resolution of $500 \mathrm{~m}$ at nadir and constant spatial sampling angle (SSA) across the swath.

To achieve this large across track coverage a mechanical scanner is employed. The axis of rotation of the scan mirror is aligned with the optical axis of the telescope (in-beam scanner), which is parallel to the velocity vector of the satellite. The normal of the scanning mirror is directed in 45 deg of the optical axis, the incident beam making an angle of 90 deg with the optical axis.

The scanner is rotating continuously clockwise about the telescope axis. The scan period of $1.729 \mathrm{~s}$ is defined by the satellite orbit and the spatial resolution.

The scanning principle also allows for regular views to dedicated calibration sources which are needed for radiometric calibration. A solar calibration device (SCAD) allows for calibration with respect to the Sun, the thermal calibration device (TCAD) is used for calibration of the thermal spectral bands, and the deep space view allows for a two point calibration of all spectral bands.

\section{Image rotation and de-rotation}

The inconvenience of a system with a static telescope and an in-beam scanner is that the rotation of the scanning mirror generates a rotation of the observed ground pixel in the focal plane, where the image rotation angle is equal to the scanner rotation angle. So, the juxtaposition of two successive scanning lines allows obtaining a regular coverage only in the center of the observed zone. To obtain a regular imaging geometry it is necessary to introduce in the optical path a system which allows for image de-rotation. The figure below shows an example for $3 \times 9$ detector elements with and without image de-rotation.
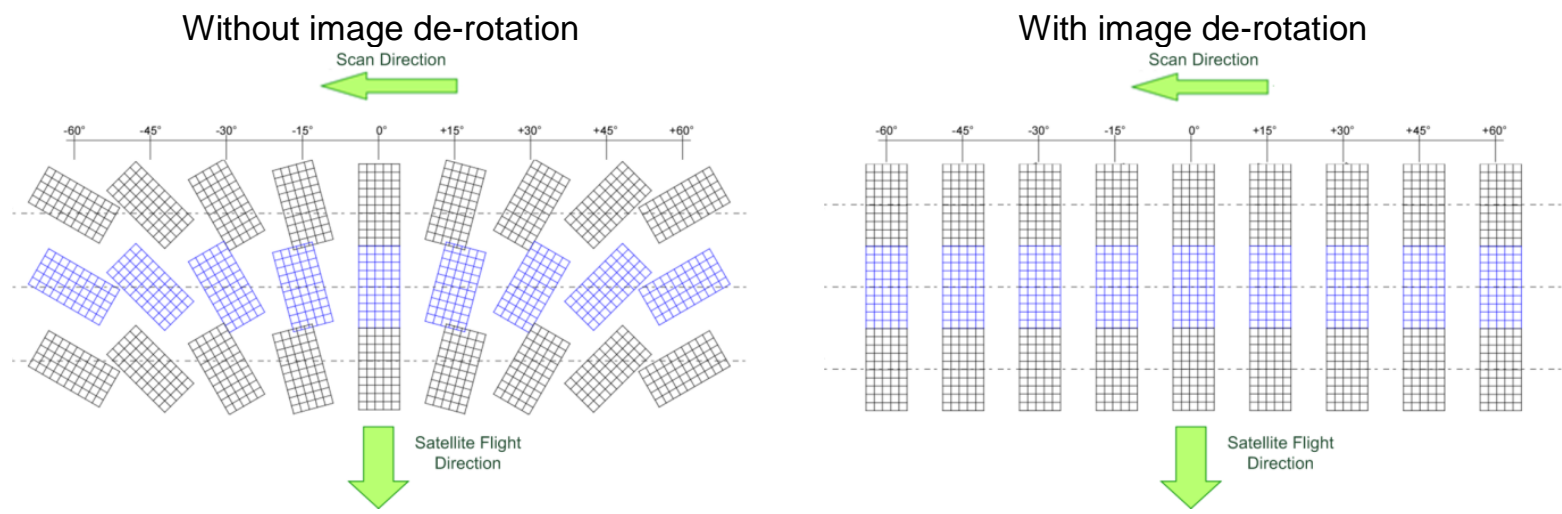

Left: Rotation of the image due to the rotation of the scanning mirror

Right: Regular image geometry achieved by image de-rotation 
A derotator is needed for physical image de-rotation, for a regular coverage as just mentioned, but also because co-registration is specified at level 0 by the customer. The rotation of an angle $\Omega$ of the derotator around the optical axis generates a rotation of the image by an angle of $2 \Omega$.

The resulting instrument concept with scanner and de-rotator is depicted in the figure below. De-rotation is achieved by a drive turning at half speed of the drive of the scanning mirror, generating a rotation of image opposite to that generated by the scanner.

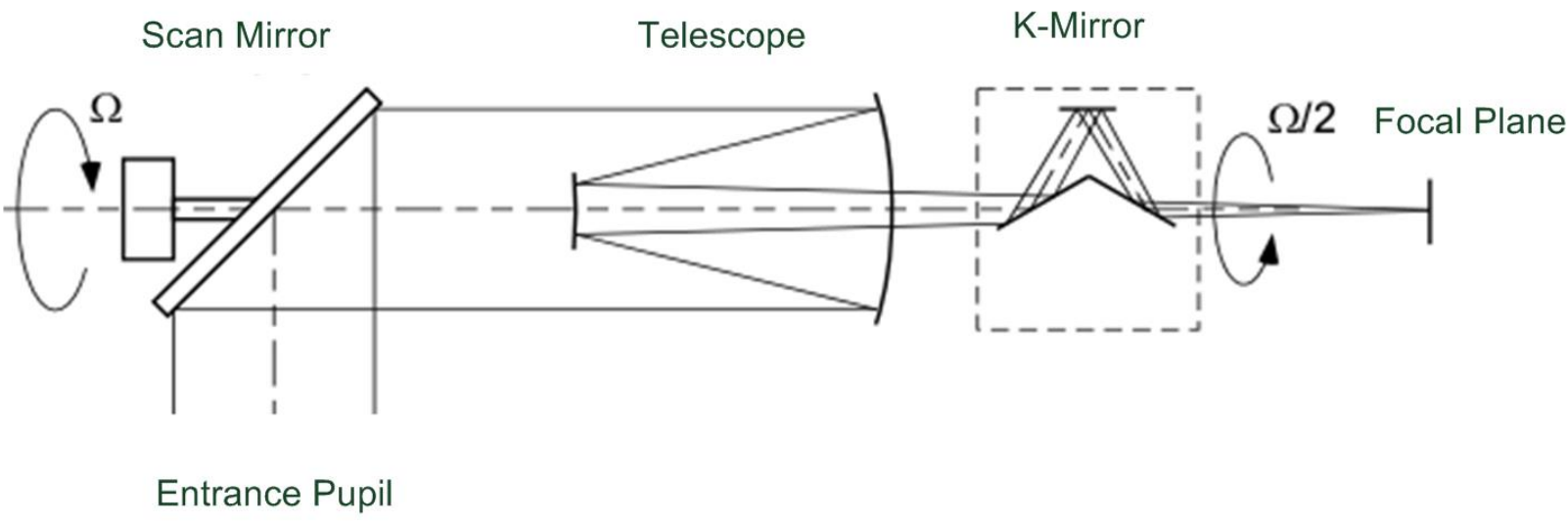

Instrument concept with scanner and de-rotator

A system for image rotation with 5 mirrors allows for reducing the level of polarisation considerably, compared to a derotator with 3 mirrors only, as described in the US patent 4929040 . With this geometry, the main axes of polarisation $\mathrm{S}$ and $\mathrm{P}$ are successively contained in the planes of the mirrors, which results at the exit in polarisation coefficients of appreciably identical values in both axes at the exit of the derotator.

\section{Spectral separation scheme}

The field of view supports the scanning of 24 ground pixel of $500 \mathrm{~m}$ resolution in flight direction and provides 10 slots for spectral channels for each of the 3 focal planes (bands). The spectral band separation between the focal planes is accommodated by 2 dichroic beamsplitters, the channel separation within each band is implemented by spectral bandpass filters. The spectral separation scheme is depicted in the figure below.

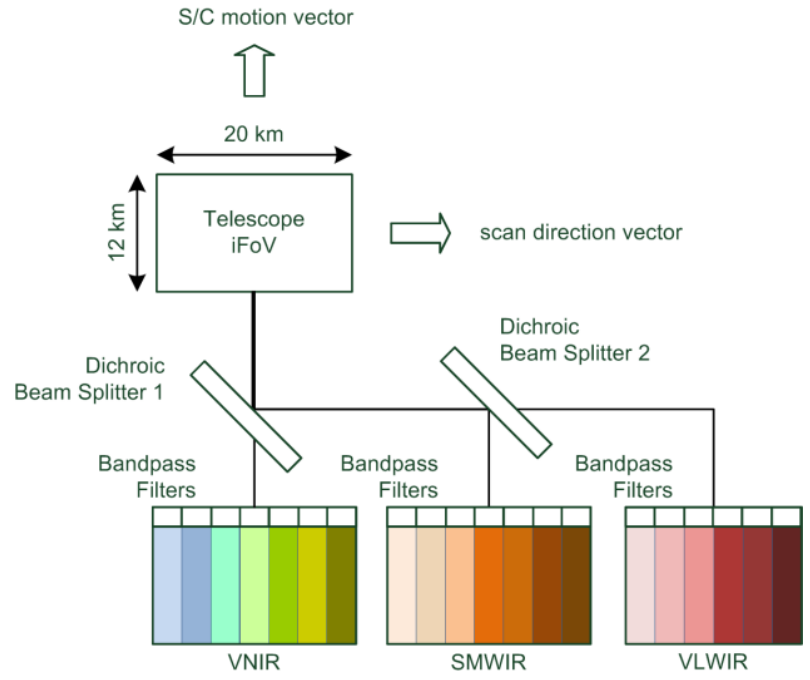

METimage spectral separation scheme 
ICSO 2020

International Conference on Space Optics

2.2 Optical architecture block-diagram

The optical architecture can be split into 8 functional units, see figure below.
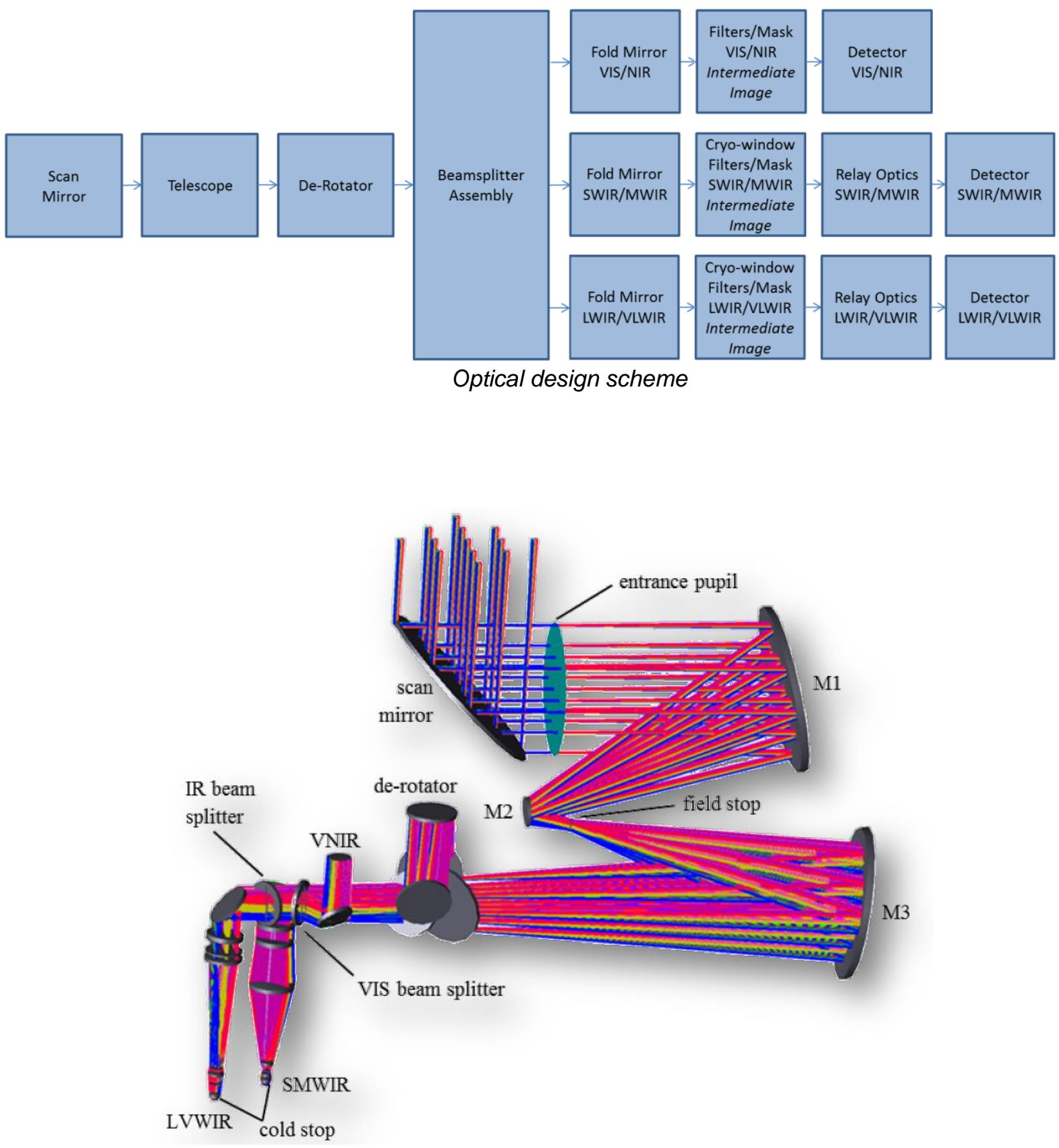

Metimage instrument optical design overview 
ICSO 2020

International Conference on Space Optics

\subsection{Description of the subsystems}

\section{$\underline{\text { Scanner mirror }}$}

The rotating scanner mirror realizes the scanning of the Earth scene. The axis of rotation of the mirror is aligned with the optical axis of the telescope. The normal of the scanning mirror is tilted $45^{\circ}$ with respect to the optical axis so that the incidental beam forms an angle of $90^{\circ}$ with the optical axis. The scanner mirror has a cylindrical radius of curvature of $760 \mathrm{~m}$ concave, in order to generate a defocus in along-track direction equivalent to the motion blur in across-track, and therefore comply with MTF homogeneity and co-registration requirements.

\section{Main characteristics: \\ - Material: Beryllium \\ - Dimensions: $260 \times 190 \mathrm{~mm}^{2}$ \\ - Optical surface: cylindrical \\ - Optical coating: enhanced protected silver \\ - Manufacturer: AMOS}

\section{$\underline{\text { Telescope }}$}

The telescope maps the input scene to an intermediate image, with a circular field of view (because of image rotation) of $\pm 0.9^{\circ}$ and focal length of $1660 \mathrm{~mm}$ at $\mathrm{F} / 10$.

The optical design is a TMA composed of 3 off-axis conical mirrors with tilts and decenters between their respective axes in order to improve performance. The main design driver was distortion, limited to a maximum of $18 \mu \mathrm{m}$ displacement of the spot over the entire field of view. The spot-radius is $20 \mu \mathrm{m}$ RMS maximum over the entire field of view.

The design was also closely driven by accommodation needs: a long back focal length for the optical path of the derotator, entrance and exit axes exactly parallel for the scanner and derotator mechanism, positions of M2 and M3 driven by envelope constraints.

Main characteristics:

- Material: Zerodur

- Dimensions: 40 (M2) to 190mm (M1) in diameter

- Optical surface: conical off-axis

- Optical coating: enhanced protected silver - Schott

- Manufacturer: AMOS

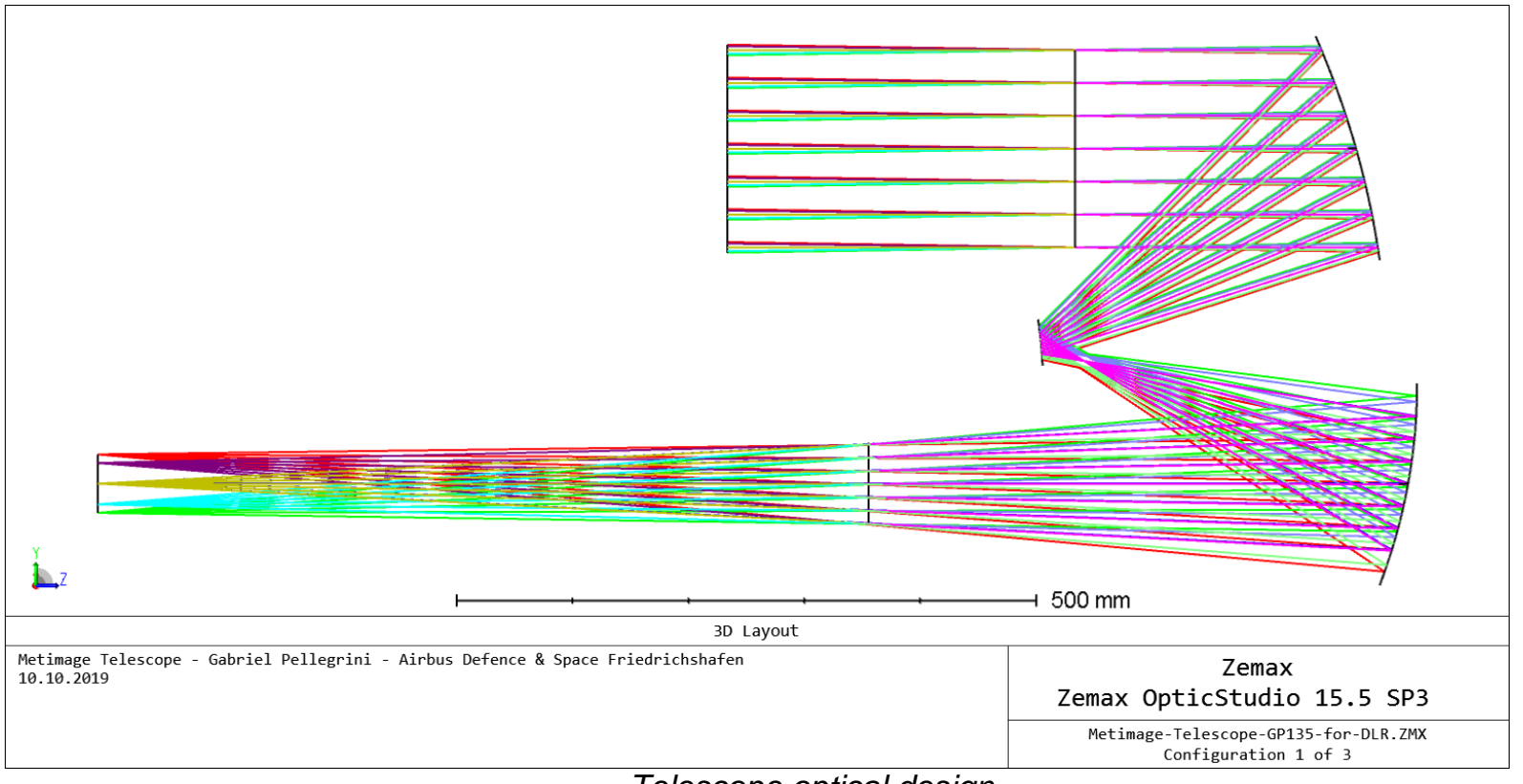

Telescope optical design 


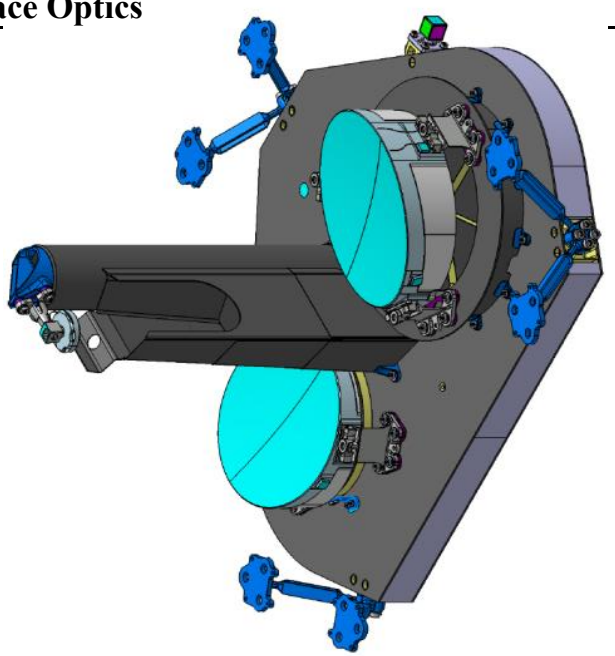

Telescope assembly mechanical design

\section{$\underline{\text { Derotator }}$}

The derotator compensates the rotation of the image introduced by the scanner mirror. It uses 5 mirrors in two perpendicular planes. The incident angles on the mirrors are arranged in such a way that the polarisation sensitivity is low. It uses $\mathrm{SiC}$ for all components in order to ensure an appropriate stability over the temperature range.

\section{Main characteristics:}

- Material: SiC

- Dimensions: 100 to $120 \mathrm{~mm}$ diameter

- Optical surface: flat

- Optical coating: enhanced protected silver - Schott

- Manufacturer: AMOS

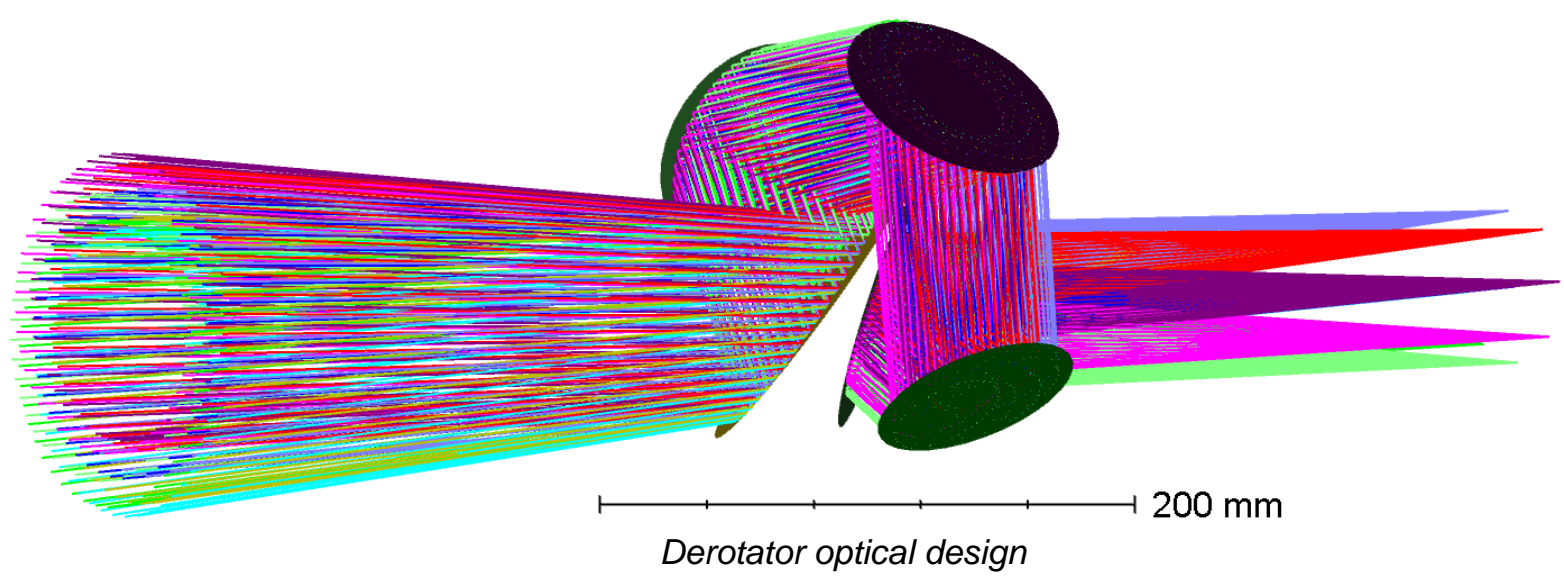

\section{Dichroic beamspitters}

The beamsplitter assembly has the function to separate the spectral range into 3 bands:

- $\quad$ VIS/NIR from $0.4 \mu \mathrm{m}$ to $0.9 \mu \mathrm{m}$

- $\quad$ SWIR/MWIR from $1.2 \mu \mathrm{m}$ to $4.1 \mu \mathrm{m}$

- $\quad$ LWIR/VLWIR from $6.6 \mu \mathrm{m}$ to $13.5 \mu \mathrm{m}$

They are flat components in ZnSe for optimal transmission in the infrared. DBS1 reflects visible wavelengths at the nominal incidence of $34^{\circ}$ and transmits the infrared, DBS2 reflects SMWIR at the nominal incidence of 
$36^{\circ}$ and transmits LVWIR. In transmission with a significant incidence angle, the parallel plates generate optical aberrations. The astigmatism generated on WFE is acceptable, and the distortion is significant but compensated on the field masks (see section 3.1).

Main characteristics:

- Material: ZnSe

- Dimensions: 70 × $50 \mathrm{~mm}^{2}$ rectangular

- Optical surface: flat

- Optical coating: dielectric coatings, dichroic on the front side and anti-reflect on the back side

- Manufacturer: Sodern - subcontractor Materion

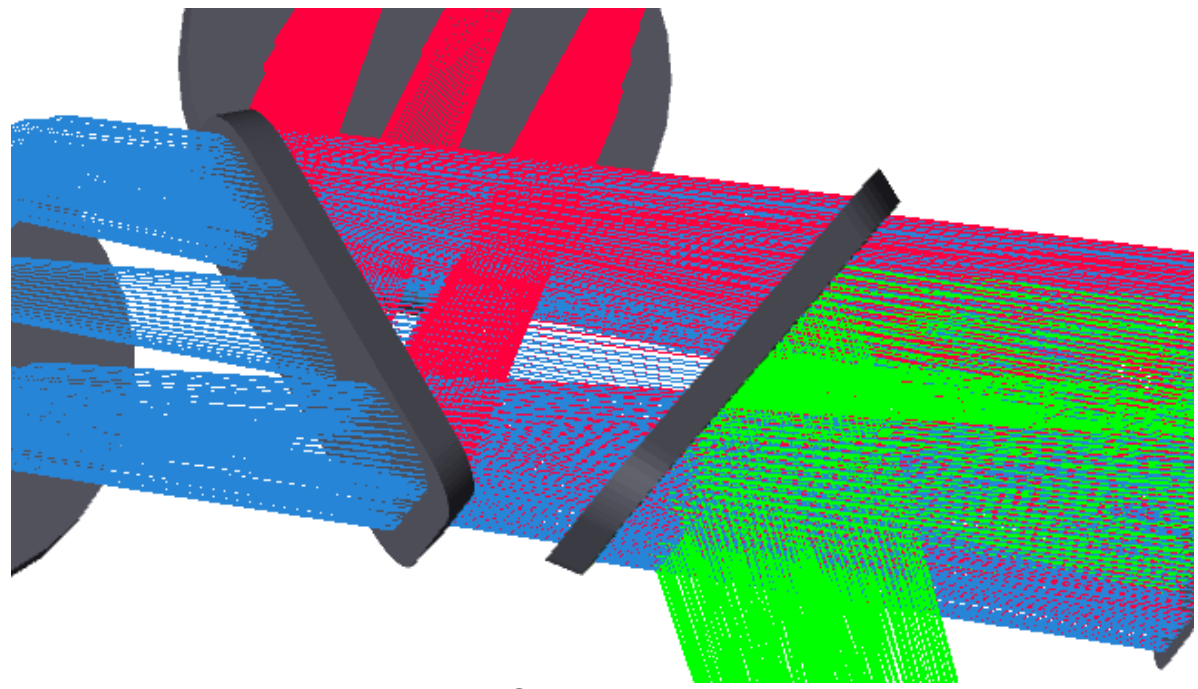

DBS optical design

\section{Folding mirrors}

There is one folding mirror at $45^{\circ}$ for each spectral band, in order to fold the beam towards the corresponding detectors, for accommodation reasons.

Main characteristics:

- Material: Aluminum

- Dimensions: $60 \times 70 \mathrm{~mm}^{2}$

- Optical surface: flat

- Optical coating: enhanced protected silver

- Manufacturer: Media Lario - Coating: Carl Zeiss
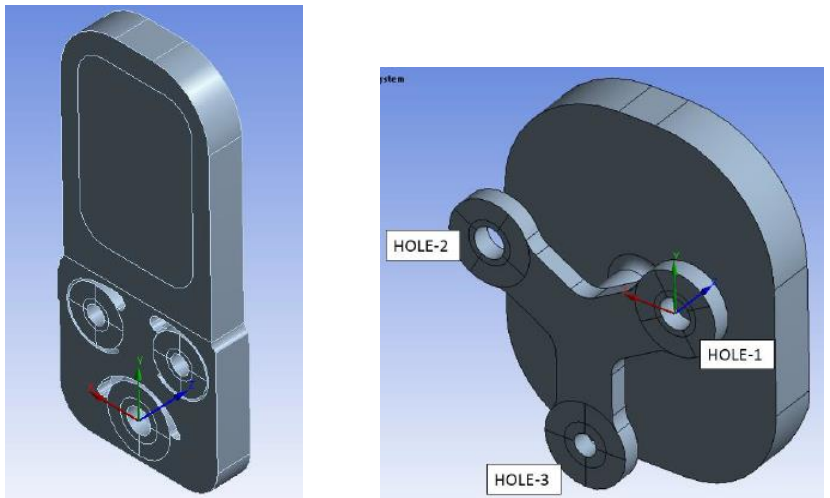

Folding mirrors mechanical design 


\section{Cryostat windows}

In IR channels, cryostat windows ensure the tightness of the cryostat for contamination and thermal reasons.

\section{Main characteristics:}

- Material: ZnSe

- Dimensions: 70mm diameter

- Optical surface: flat

- Optical coating: dielectric anti-reflective coating on both sides

- Manufacturer: Schott Suisse SA Yverdon

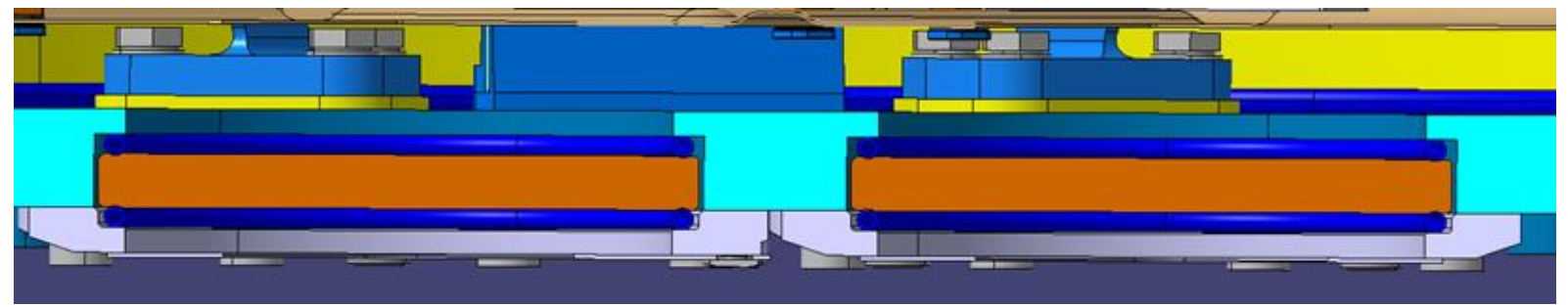

Cryostat windows mechanical mounting

\section{Optical filters}

Three focal planes correspond to the three spectral bands:

- VNIR filters and detector

- IR filters and field mask assembly for IR channels, as intermediate focal plane.

\section{Main characteristics:}

- Material: BK7 for VNIR, Sapphire for SMWIR, Germanium for LVWIR

- Dimensions: $20 \times 24 \mathrm{~mm}^{2}$ for VNIR, $40 \times 24 \mathrm{~mm}^{2}$ for IR

- Optical surface: flat

- Optical coating: dielectric band-pass and AR coating

- Manufacturer VNIR: Sodern - Coating: OBJ

- Manufacturer IR: Hensoldt - Coating: VIAVI

\section{Relay optics}

In the two IR bands, a combination of refractive optics reduces the image size by a magnification of 0.135 in order to be compatible with the dimensions of the infrared detectors. These Relay optics consist of lenses operating in cryogenic temperature, are about $180 \mathrm{~mm}$ long, and include a cold stop in order to block all direct light coming from the warm structure. The manufacturer for this subsystem is Hensoldt, subcontracting to IIVI. The optical design is made by Carl Zeiss.

The SMWIR system consists of 4 aspheric lenses in ZnSe and Si. Lens 2 and 4 are aspheric on both sides in order to compensate the system's aberrations. The first lens is tilted to work against the chromatic pupil variation. The axial chromatic shift is compensated by a $1.7^{\circ}$ tilted detector. 


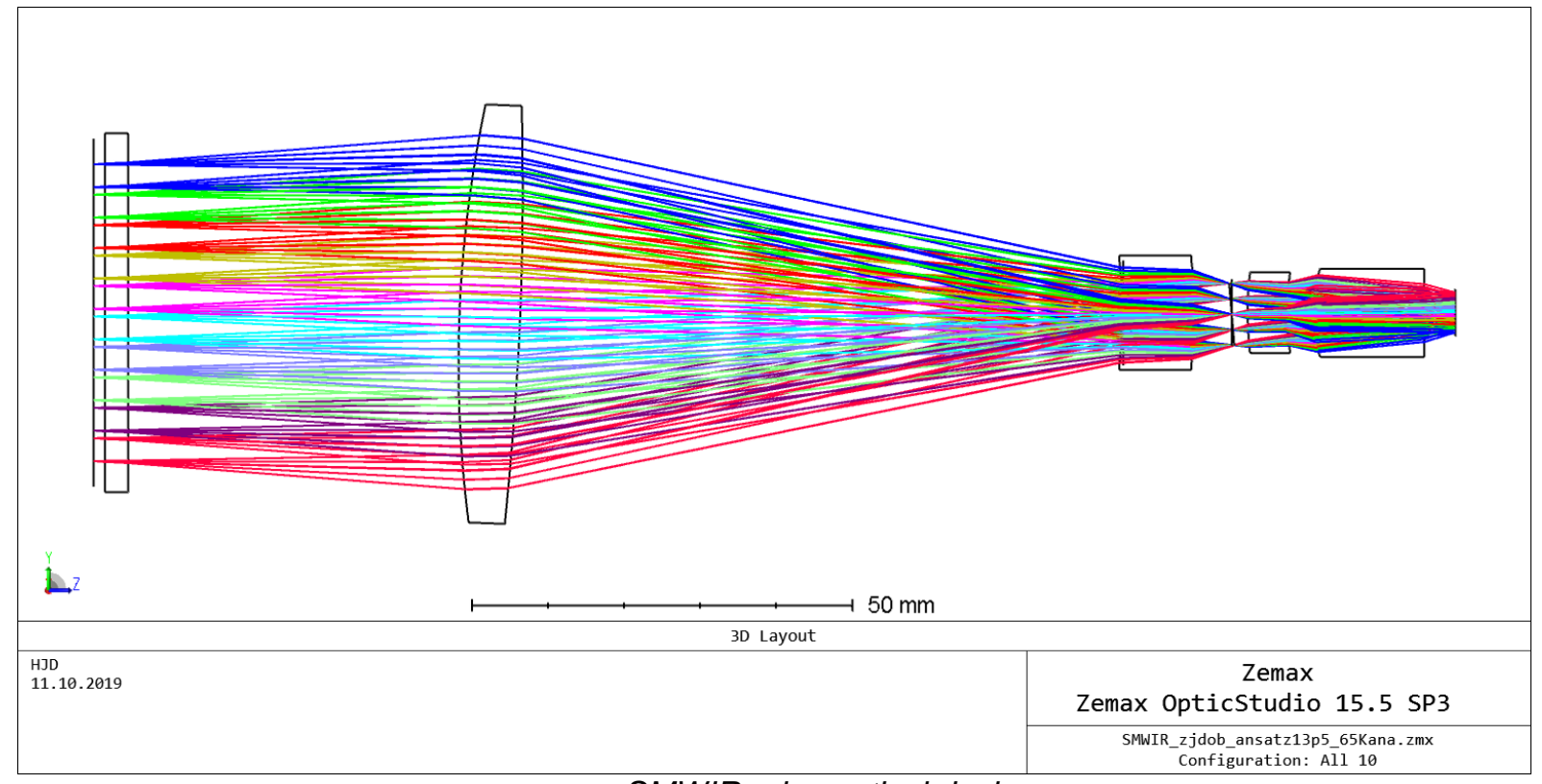

SMWIR relay optical design

The LVWIR system consists of 3 thin Ge lenses. Lens 2 has an aspheric surface. For LVWIR, lenses, cold stop and detector are rotationally symmetric around the optical axis, since chromatism is reduced in this wavelength range.

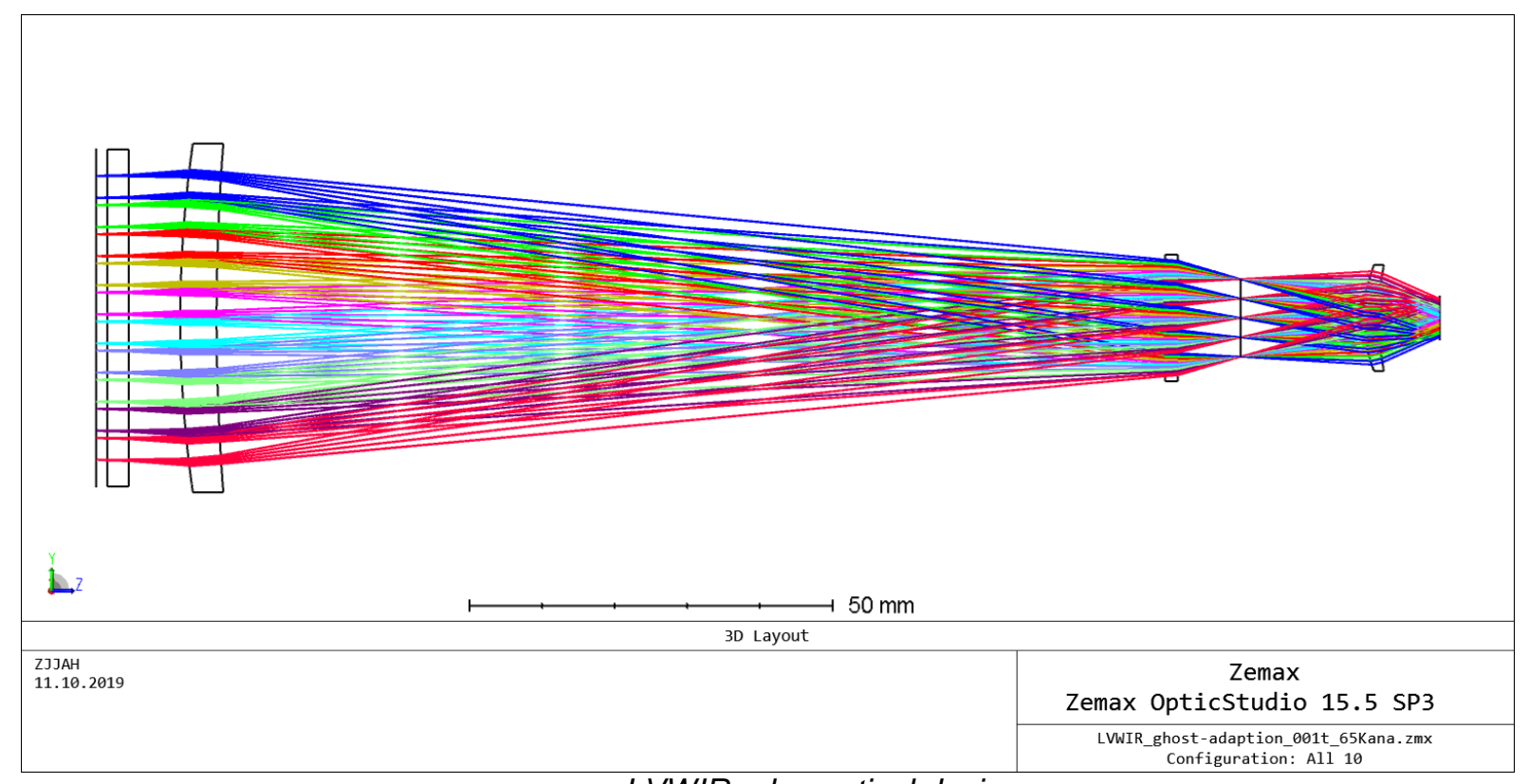

LVWIR relay optical design 


\section{OPTICAL DESIGN DRIVERS}

\subsection{Co-registration}

The co-registration requirement is a driver for distortion of the instrument and imposes the use of field masks for the infrared channels. As mentioned in section 2.1, the specification of co-registration at level 0 already had a strong impact on the architecture of the instrument, with the implementation of the derotator to physically correct the image rotation linked to the scan principle.

\section{Field masks}

Physical masks are placed at the intermediate focal plane of the infrared channels in order to ensure a good correlation between the three focal planes: VNIR, SMWIR and LVWIR. In these planes, the magnification is identical. The masks contain $1 \mathrm{~mm}^{2}$ holes that represent the pixels for the infrared channels. The aberrations of the relay optics, placed after the field masks, are therefore not contributing to the co-registration budget. Another advantage of the field masks is to compensate for the distortion of the beamsplitters, as described below.

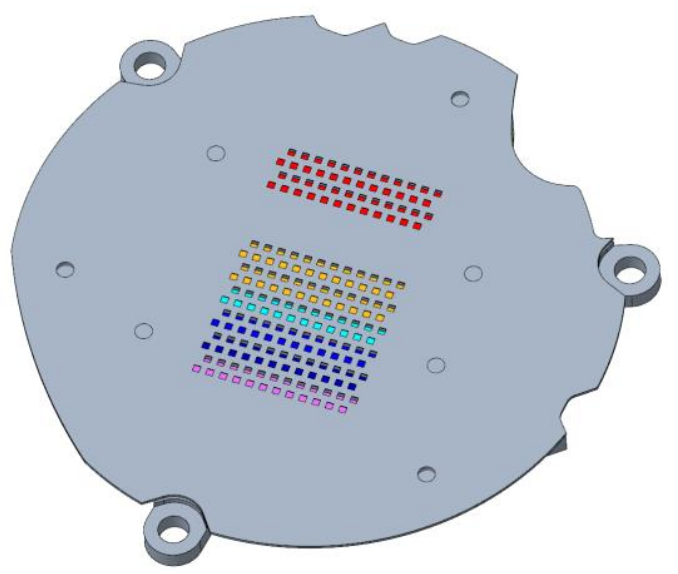

Field mask in LVWIR focal plane

\section{Distortion}

As mentioned, distortion has to be calculated in the intermediate image (VNIR detector and IR field masks). The distortion of the relay optics does not enter into the co-registration requirement thanks to the field masks.

Distortion is mainly generated by the telescope and the beamsplitters. The telescope design has a distortion lower than $18 \mu \mathrm{m}$ over the entire circular field of view of $\pm 0.9^{\circ}$, as mentioned in section 2 .

The beamsplitters generate distortion because they are tilted with incidences of $34^{\circ}$ and $36^{\circ}$ with respect to the optical axis. This distortion amounts up to $26 \mu \mathrm{m}$. This distortion is higher than the one introduced by the telescope and would dominate the co-registration budget. In order to limit its effect, the pattern of the field masks is adjusted to follow the distortion pattern ( $26 \mu \mathrm{m}$ maximum deviation to a regular pattern). As a result, the field masks sample the distorted image generated by the beamsplitters and the impact on co-registration is thus minimized.

It also has to be noted that the distortion of the beamsplitters couldn't be compensated by the telescope, since the image rotates with scan angle at this stage, but not at beamsplitters level, because they are placed after the derotator. It could be compensated by a wedge angle and cylindrical radii of curvature on the folding mirrors, but this solution has been judged too complicated and not attractive compared to the adjustment of the field masks pattern. 
ICSO 2020

\subsection{Pupil imaging}

\section{$\underline{\text { Pupil budget }}$}

The pupil budget is a crucial parameter to handle. Pupil planes must be aligned as well as field planes in order to avoid losing signal through the instrument. Besides, it is very important for straylight purposes, that the cold stops in the relay optics block all direct light from the warm structure.

All contributors of this pupil budget are carefully handled via design constraints and tolerancing on manufacturing and alignment tolerances, and measurement accuracies.

The most dominant contributors are:

- Telescope tolerances between the entrance aperture and the exit pupil.

- Relay optics optical design with a particular focus on pupil imaging through wide spectral ranges.

- Relay optics position and diameter of the cold stops in cryogenic environment, in order to generate an entrance pupil matching with the exit pupil of the telescope.

- Characterisation of the pupil planes and alignment of the subsystems at instrument level.

- On top, management of the derotator alignment and stability in order to minimize the wobble effect of the pupil, i.e. its movement through scan.

\section{$\underline{\text { Pupil diffraction }}$}

Due to the presence of the field masks with $1 \mathrm{~mm}^{2}$ apertures in the infrared intermediate image plane, a diffraction effect occurs for the pupil between the exit pupil of the telescope and the cold stop plane in the relay optics. This effect is particularly large in LVWIR for longer wavelengths.

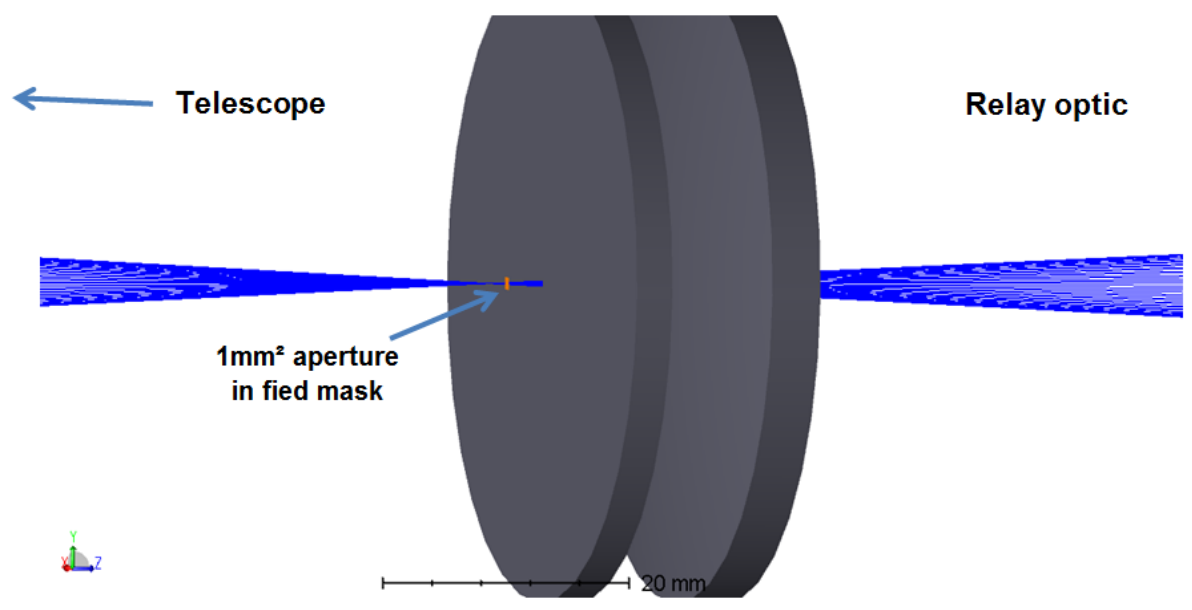

Origin of the pupil diffraction effect

This effect is important to be taken into account, since it degrades SNR:

- Some useful signal is diffracted out of the useful beam and lost.

- The cold stops must be slightly downsized in order to avoid most of the diffracted warm radiation from the structure.

The pupil diffraction effect has been calculated in Python and OpticStudio (in Physical Optics Propagation mode), in order to compare the results. An excellent correlation has been found, here below the comparison of the intensity distribution in the cold stop plane, for one centered field point at $\lambda=12 \mu \mathrm{m}$ : 

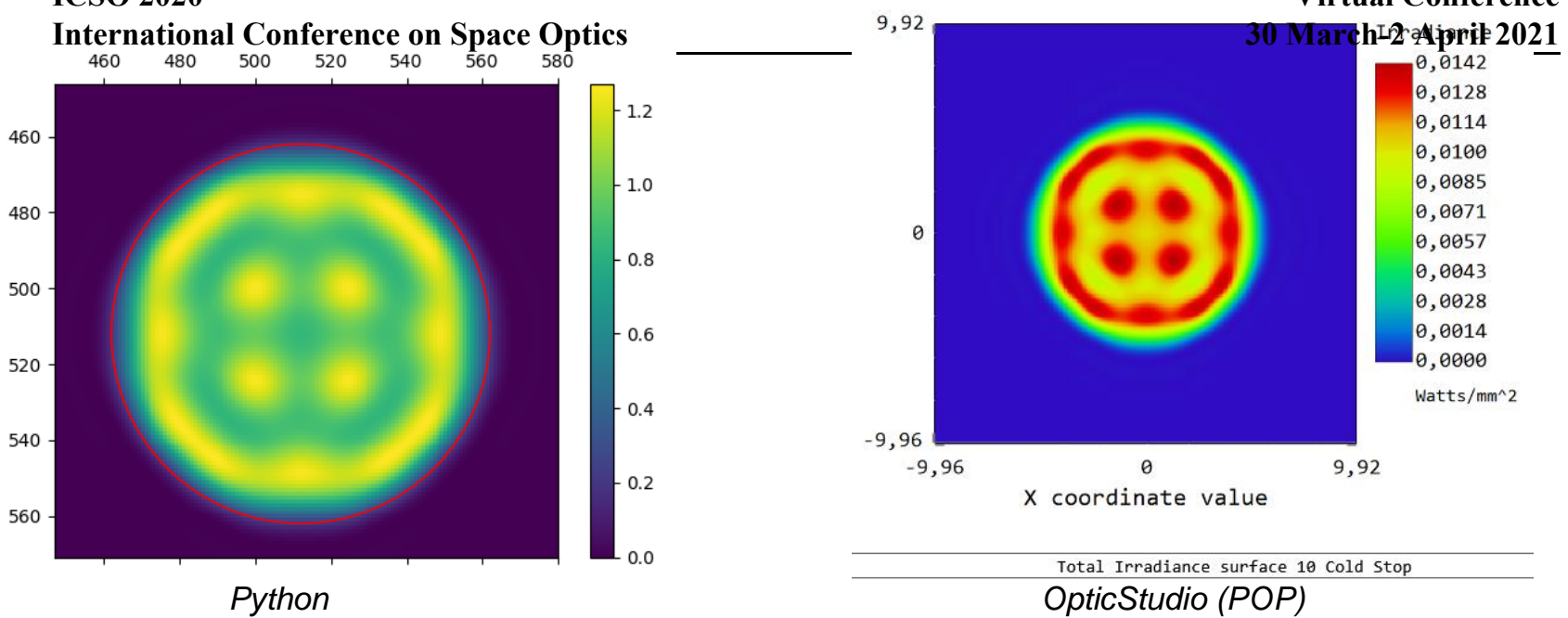

Now considering a full illumination in a $1 \mathrm{~mm}^{2}$ hole at $\lambda=12 \mu \mathrm{m}$ from Python, the intensity distribution in the cold stop plane has the following shape:
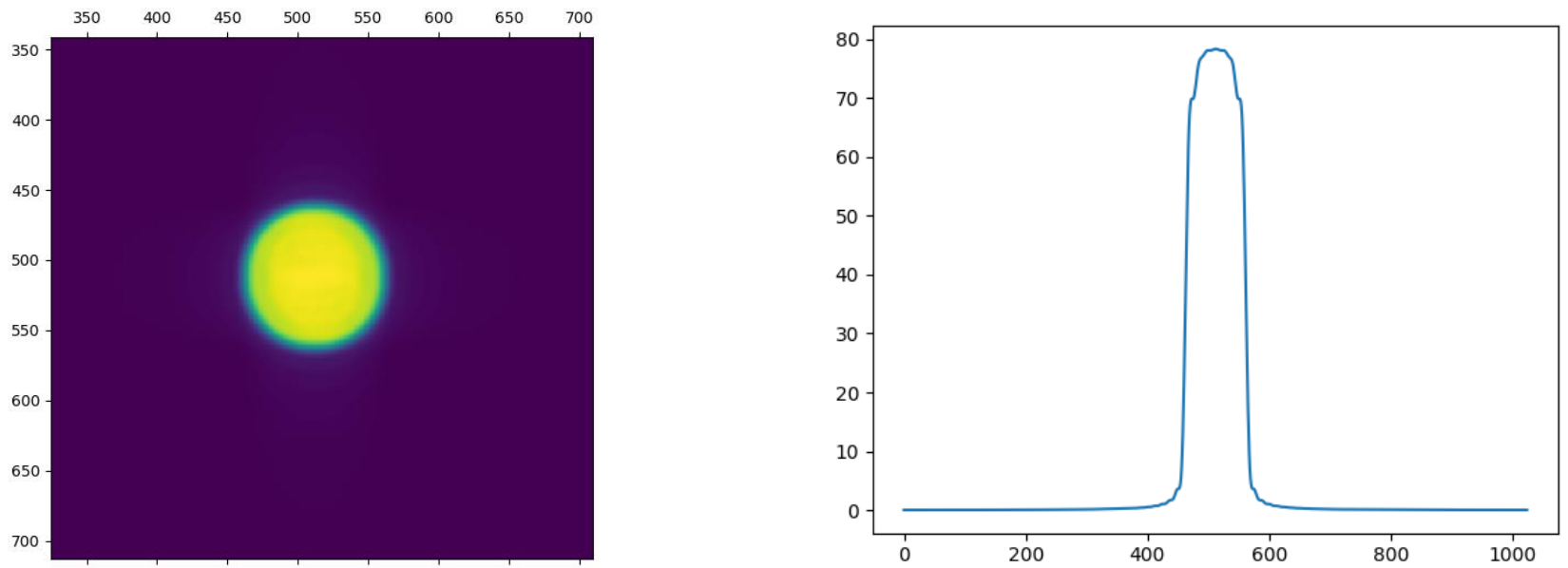

This effect has been integrated into the performance models, and led to a reduction of the cold stop diameter by $10 \%$ compared to the one based on the geometric propagation of the rays.

\subsection{Straylight}

\section{Baffling principle}

Straylight control is first ensured by appropriate baffling. To this aim, the following baffles have been designed:

- Entrance baffle to limit out-of-field light entering the system. Due to the scanning principle, at this level this goal is achieved mainly in along-track direction only.

- A physical entrance stop after the scanner mirror.

- A field stop at the intermediate image after M2 of the telescope, enabling to reduce further out-of-field light into the rest of the instrument. This was a constraint for the optical design of the TMA telescope.

- Derotator housing.

- Additional baffles after the derotator, with the particular goal to avoid illuminating reflective surfaces, e.g. metallic mounting surfaces.

- Field masks, reducing the beam to the useful light only, ensuring a minimum radiation is entering the cryostat.

- Cold stops inside the infrared relay optics, as mentioned earlier, to block warm radiation and define the infrared pupils. 
The tight straylight requirements lead to stringent specifications on all optical components in terms of:

- State of the art surface roughness requirements on all components.

- Surface defects (scratches and digs), and their effect on straylight calculated on the basis of available literature.

- For refractive components: bulk defects (bubbles and inclusions) and selection of high quality grades of material.

- Cleanliness specified at component level, and budgeted for all steps throughout AIT.

\section{Ghosts}

Ghosts linked to unwanted reflections on refractive surfaces led to specific constraints on the optical design (adjustment of material, thickness or wedge) and coatings (monitoring of the surface reflection in order to reduce the intensity of the ghost).

On the Metimage instrument, the following ghosts have been handled:

- Beamsplitter ghosts: the anti-reflective coatings on back surfaces have been designed for maximum transmission, but also with the goal to reduce ghosts, leading to some additional constraints on the coating design.

- Cryostat windows ghosts: these components are placed close to the infrared field masks, and are therefore prone to generating ghosts. An early trade-off has been realized in order to define the properties of the components.

- Relay optics ghosts: the relay optics being placed after the infrared filters, ghosts are particularly important since they generate cross-talk between the channels. Again, ghosts must be taken into account in the optical design phase. A fine-adjustment of the radii of curvature is enough to defocus the ghosts, at the cost of a minor adaptation of the design. The choice of materials can also contribute to the blocking of out-of-band wavelengths. Ultimately, residual ghosts can be reduced by image post-processing.

\subsection{Polarisation sensitivity}

\section{Scanner and derotator designs}

The requirements on polarisation sensitivity and its homogeneity over scan were taken into account in the design phase, by the choice of the scanner and derotator designs, as described in section 2.1:

- In-beam scanner: this scanning principle enables to have a constant incidence angle of $45^{\circ}$ on the mirror, independently of the scan angle.

- 5 mirrors derotator in order to limit the effect on polarisation sensitivity.

This had to be carefully handled during the design phase, particularly wrt the accommodation of the derotator in a limited envelope, and the impact of its optical path length on the back focal length of the telescope. Also, the large number of mirrors brings other constraints on the optical coating (transmission), scattering characteristics (roughness and contamination) and stability: to this effect, the derotator is completely made of $\mathrm{SiC}$ as described in section 2.3.

\section{Optical coatings}

Due to the large number of optical components in the instrument, polarisation sensitivity must be reduced as much as possible on every optical interface. This has led to specific coating designs for all components, in particular:

- Scanner, telescope, derotator and folding mirrors (10 mirrors in total): enhanced protected silver with additional dielectric layers developed by Schott and Carl Zeiss. Specific care taken for mirrors working at $45^{\circ}$ angle of incidence. 
- Beamsplitters: Dielectric coatings on ZnSe working at $35^{\circ}$ angle of incidence over a very wide spectra range, developed by Materion with minimal polarisation sensitivity as design driver.

The optical analysis shows a partial compensation between components acting in perpendicular planes in VNIR and SMWIR spectral ranges, however the LVWIR spectral channels being in transmission through both beamsplitters, polarisation sensitivity is additive and therefore higher, which is consistent with the top-level requirements.

\section{DEVELOPMENT STATUS}

Instrument CDR has been declared successful at the beginning of 2020 , and confirmed the maturity of the design in all disciplines, performance budgets and AIT approach.

Manufacturing of subsystems and components has been launched well in advance, after PDR, as soon as the technical maturity at lower level was considered sufficient, for schedule reasons. At beginning of 2021, the simplest optical components have been delivered and the other subsystems expect a soon delivery of the first optical model.

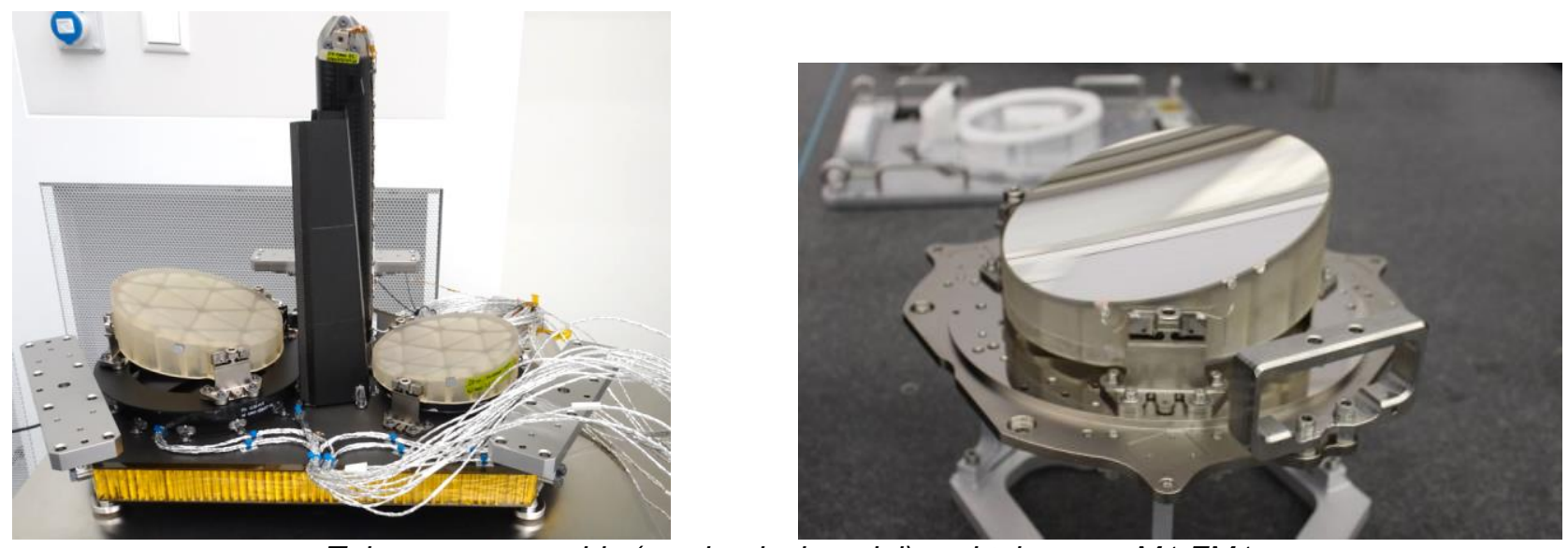

Telescope assembly (mechanical model) and telescope M1 FM1
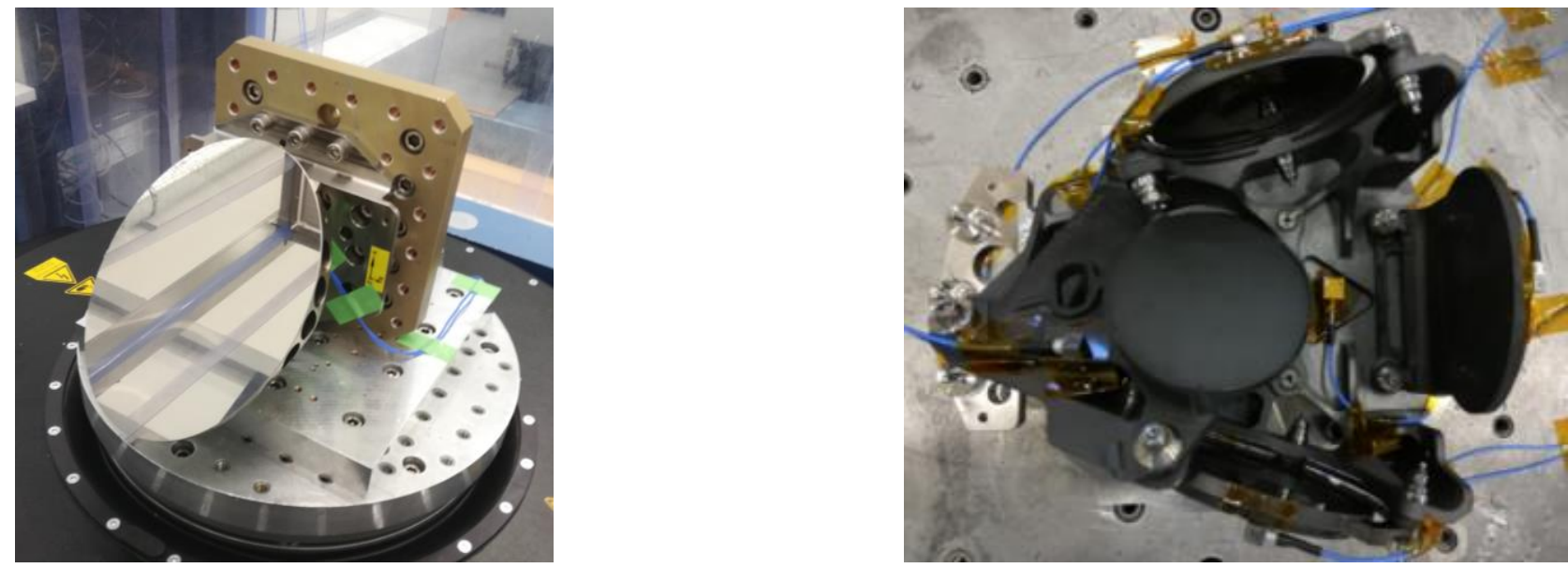

Scanner (engineering model) and derotator (mechanical model) 


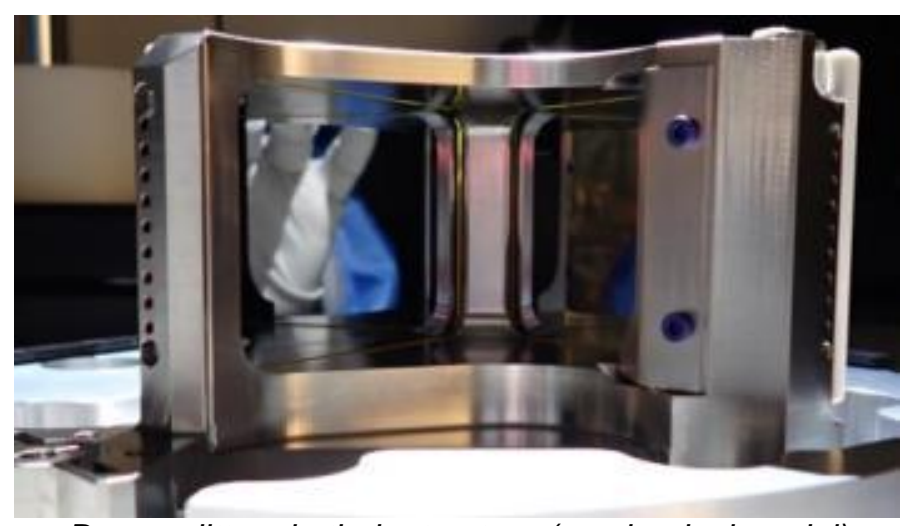

Beamsplitters in their structure (mechanical model)
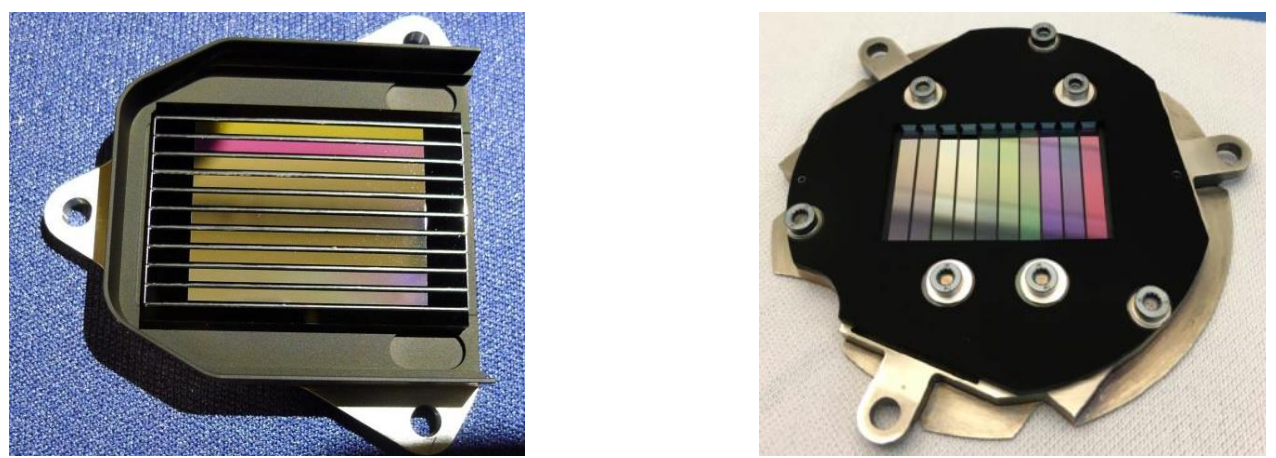

VNIR and LVWIR filters (FM1)
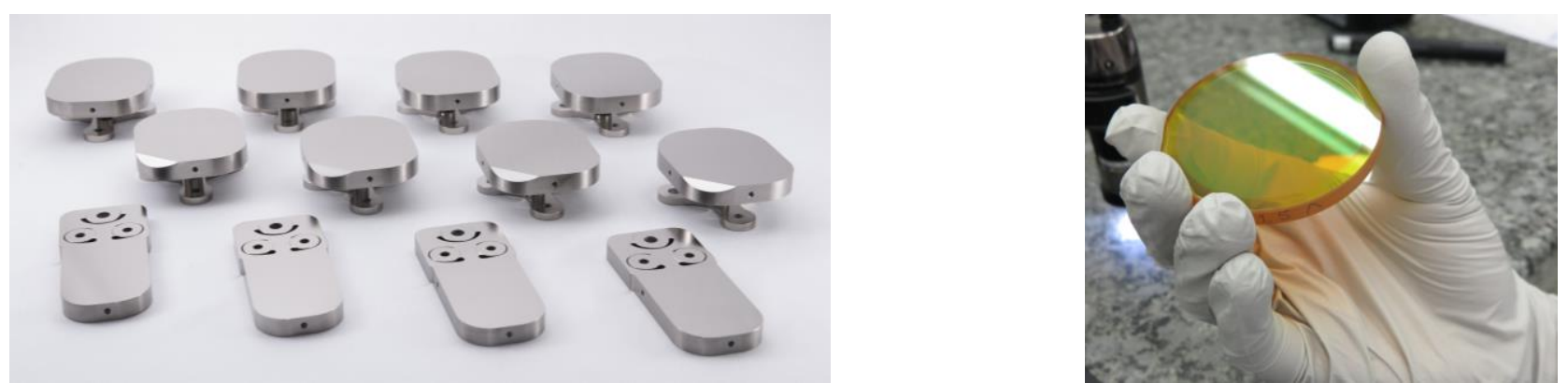

Folding mirrors (all models) and SMWIR cryostat window (FM1)

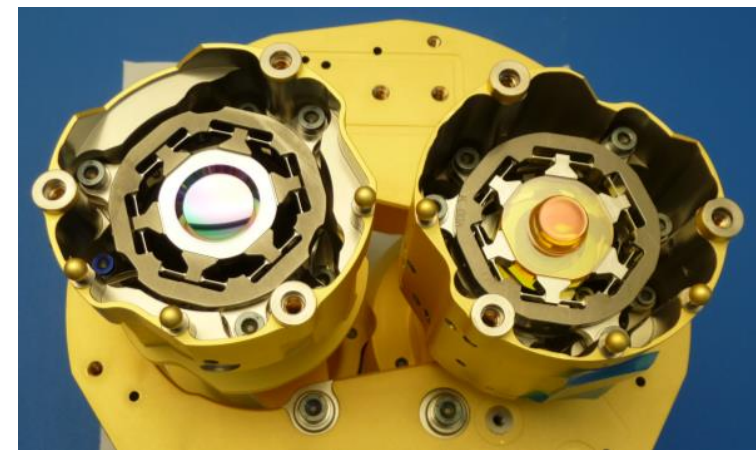

Relay optics (FM1)

\section{ACKNOWLEDGEMENT}

The work described was performed on behalf of the German Space Administration with funds from the German Federal Ministry of Transport and Digital Infrastructure and co-funded by EUMETSAT under DLR Contract No. 50EW1521: 